\title{
Catástrofes de la Educación o la Debacle que no Cesa: La Innovación como Lucha contra los Elementos
}

\section{Catastrophes of Education or the Endless Debacle: Innovation as a Fight against the Elements}

\author{
Juan Manuel Moreno * \\ Banco Mundial \\ UNED, España
}

DESCRIPTORES:
Crisis del aprendizaje
Escolarización
universal
Educación en la
sombra
Innovación educativa
Recesión de la
democracia

\begin{abstract}
RESUMEN:
Tanto en los países con los sistemas educativos más desarrollados como, desde luego, en aquellos con los más frágiles, la educación tiende a percibirse como una suerte de catástrofe, como un sector fallido y atrasado casi por definición al que todos reclaman cambios profundos. Este artículo trata primero de identificar cuáles son las auténticas catástrofes de la educación -la persistente crisis de aprendizaje, la recesión de la democracia y sus efectos sobre la universalización de la educación, y el imparable ascenso de la educación en la sombra- que son anteriores a la pandemia de 2020 y a sus también catastróficas consecuencias. Estas catástrofes hacen de la innovación educativa una lucha contra los elementos y contribuyen a explicar por tanto las paradojas, desafíos y dificultades del desarrollo y el progreso de la educación y el aprendizaje en nuestro mundo. A continuación, y a partir de ese análisis de tendencias globales, se presenta evidencia seleccionada sobre las direcciones más sólidas de innovación en educación con un foco en los países en desarrollo. Las agencias de desarrollo, bilaterales y multilaterales, las fundaciones, ONGs, y otros actores que invierten miles de millones de euros en la educación de estos países, necesitan con urgencia esa evidencia para no malgastar sus recursos.
\end{abstract}

\section{KEYWORDS:}

Learning crisis

Universal schooling

Shadow education

Educational innovation

Democratic recession

\begin{abstract}
:
In both developed and emerging education systems education tends to be socially and politically perceived as a failed and backward sector that requires a radical overhaul and cutting-edge innovation. This paper sets out to identify the true catastrophes of education -the persistent crisis of learning, the democratic recession and its impact on universal education, and the rampant growth of shadow education-which precede the 2020 pandemic and its equally catastrophic consequences on the sector. These socalled catastrophes allow an analysis of the paradoxical challenges that education development is facing nowadays. The paper then ends with a selection of available evidence on the impact and cost-effectiveness of innovative interventions that could lead to a much-needed global agenda for education innovation, particularly for developing countries. The evidence available is increasingly compelling and sophisticated. Development agencies, bilateral and multilateral, foundations, NGOs, and many other actors who spend billions of dollars in the education systems of these countries, urgently require such evidence so that those resources are not squandered.
\end{abstract}




\begin{abstract}
...el atractivo de las ideas regresivas es perenne, y no debemos cejar en la defensa de la razón, la ciencia, el humanismo y el progreso. Cuando no acertamos a reconocer nuestro progreso logrado a duras penas, podemos llegar a creer que el orden perfecto y la prosperidad universal son el estado natural de las cosas, y que todo problema es un ultraje que requiere culpar a los malhechores, derribar las instituciones y empoderar a un líder que restablecerá la grandeza legítima del país. (Pinker, 2018, p. 547)
\end{abstract}

\title{
1. Introducción
}

A medida que el planeta se acerca a la escolarización universal y que la duración de la llamada educación básica ha pasado desde cuatro hasta al menos diez años en prácticamente todos los países del mundo, el desencanto con la educación, las expectativas frustradas y la politización del sector educativo aumentan por doquier. Los motivos por los que esto ocurre son obviamente distintos dependiendo de la posición ideológica que se adopte, lo que también es cierto en cuanto a los motivos por los que, sea cual sea la sensibilidad política, todo el mundo se apunta a la retórica de que la educación "es el futuro", la inversión -pública y privada- "más estratégica", y la "única esperanza" para el progreso y la supervivencia de personas, comunidades, países y hasta de la especie humana; y así se va extendiendo la brecha entre expectativas siempre al alza y resultados tenazmente percibidos como decepcionantes o inaceptables. Con ello, el sector educativo se mantiene como crucial en la retórica, pero muy débil en la práctica política y en la opinión pública. Esta debilidad política y mediática se presenta con frecuencia a través de mensajes enfatizando que la educación está en plena decadencia y en permanente peligro. Son mensajes que resumen con bastante eficacia la batalla ideológica en materia de educación: el responsable de todos los males sería para empezar el Estado (para unos porque se desentiende de la educación y para otros porque quiere monopolizarla) aunque también lo sería el Mercado (para unos por mercantilizar el derecho a la educación y para otros por no ser lo suficientemente dinámico para responder a la demanda de servicios educativos). En todo caso, el resultado es que no existe otro sector con tanta y tan diversa presión -política, social y económica- por reformar e innovar.

Este escenario enquistado se aceleró en 2020 por los efectos de la pandemia del Covid 19, que ha supuesto la repentina irrupción de una auténtica distopía educativa en todo el mundo. Si la presión por innovar era ya grande, el cierre de escuelas durante meses y la perspectiva de un largo periodo de escolarización híbrida han urgido aún más la búsqueda de piedras filosofales, bálsamos de fierabrás y balas de plata. Con la mejor intención, muchos se han apresurado a bendecir la nueva situación como una oportunidad histórica para un "renacimiento educativo" o para "reconstruir" de raíz la escolarización. Sin negar que pueda ser así eventualmente, lo que es seguro es que, en el corto y medio plazo, se acelera y se expande la desconexión entre expectativas que siguen subiendo y realidades tozudas que las seguirán defraudando.

En definitiva, tanto en los países con los sistemas educativos más desarrollados como, desde luego, en aquellos con los más frágiles, la educación tiende a percibirse como una suerte de catástrofe, como un sector fallido y atrasado casi por definición al que todos reclaman cambios profundos. Este artículo trata primero de identificar cuáles son las auténticas catástrofes de la educación -anteriores ya a la pandemia de 2020 y a sus también catastróficas consecuencias- que hacen de la innovación educativa una lucha contra los elementos y contribuyen a explicar por tanto las paradojas, desafíos y dificultades del desarrollo y el progreso de la educación y el aprendizaje en nuestro mundo. A continuación, y a partir de ese análisis de tendencias globales, presento evidencia seleccionada sobre las direcciones más sólidas de innovación en educación.

\section{Catástrofes y debacles en educación}

\subsection{La persistente crisis del aprendizaje}

En esencia, la llamada crisis del aprendizaje es la brecha creciente entre años de escolarización y el aprendizaje que realmente consiguen los estudiantes en esos años. Mientras que la media de años de escolarización se ha doblado y en algunos países incluso triplicado en pocas décadas, el aprendizaje permanece 
estancado para una proporción muy alta de estudiantes en la mayor parte del mundo. Se democratiza el acceso, aumenta el gasto educativo, se universaliza la educación básica y crece de modo espectacular la matrícula en educación infantil y en educación superior, pero proporciones inusitadas de alumnos continúan dejando la escuela sin ni siquiera saber leer y escribir correctamente en su propio idioma.

En 2019, el 48 por ciento de los estudiantes de 10 años en el mundo no era capaz de hacer una lectura comprensiva de un texto breve. En los países de bajo ingreso, los más pobres, la cifra se eleva hasta el 90 por ciento de los estudiantes; baja al 55 por ciento en el grupo de los de ingreso medio-bajo, al 29 por ciento en los de medio-alto, y al 9 por ciento en el pequeño club de los países ricos (Azevedo et al., 2021). La existencia de este porcentaje de estudiantes que no es capaz de leer y entender un texto breve ajustado a su edad se ha bautizado recientemente como "pobreza en el aprendizaje". Si bien es cierto que el concepto tiene un sesgo claramente economicista, y que los resultados de la escolarización no pueden medirse ni juzgarse exclusivamente en función del aprendizaje de la lectura comprensiva, la elección de este indicador se justifica, primero, porque la lectura es la llave que abre las puertas a muy buena parte de los aprendizajes ulteriores y, segundo, porque es un indicador fácil de entender y relativamente fácil de medir. Además, poner nombre a los problemas, en este caso a la pobreza extrema de aprendizaje, permite colocarlos en el mapa del debate público global y, con ello, es posible también ponerles número, es decir, obtener y presentar evidencia sobre el volumen y las dimensiones del problema -el espacio que ocupa en ese mapa-, que hasta ahora no se había podido sacar de la invisibilidad.

A la vista de esos números, tan dolorosos como inaceptables, cabe poca duda de que se trata de una catástrofe educativa global que no tiene salida fácil ni desde luego inmediata. Al contrario, la evidencia disponible sobre el impacto del cierre de escuelas durante la pandemia de 2020-2021 apunta claramente a pérdidas de aprendizaje muy notables, especialmente en los países más pobres y entre los estudiantes social y económicamente vulnerables de todo el mundo (United Nations, 2020). En otras palabras, la pobreza en el aprendizaje va a aumentar en los próximos años, y tanto más cuanto más tiempo estén cerradas las escuelas. Además, en el contexto de la crisis económica provocada por la pandemia, con claras consecuencias sobre el gasto en políticas sociales incluida la educación, cabe plantear que podemos estar ante el primer retroceso global de la expansión del acceso a la educación (el efecto en este sentido ha sido inmediato y muy fuerte sobre los periodos no obligatorios, i.e., Infantil y Superior) y tal vez ante la primera generación de estudiantes en muchos países que tendrá menos oportunidades educativas que la generación anterior.

\subsection{Universalización de la educación básica y recesión de la democracia}

Es bien sabido que la democracia como modelo de convivencia y de organización del Estado, con su separación de poderes y reconocimiento de las minorías, ha sufrido muchos y muy serios retrocesos a lo largo de su corta Historia. Después de la Segunda Guerra Mundial, y especialmente a partir de los años 60, se produjeron simultáneamente dos procesos de fuerte expansión, el de la democracia, por un lado, y el de la educación básica, por otro. En el año 2000 se estima que el $63 \%$ de la población mundial vivía en países democráticos. Pocos años después, hacia 2008 (inmediatamente antes de comenzar la Gran Recesión), la escolarización universal en primaria era ya un objetivo prácticamente conseguido. Y el mundo ya tiene como objetivo para 2030 la universalización de la educación secundaria.

La universalización de la educación, en términos de expansión del acceso tanto a la educación básica como a la no obligatoria, ha continuado con fuerza hasta 2020 a pesar de la Gran Recesión de 2008 a 2015. Sin embargo, el retroceso de la democracia, al que hoy conocemos como "recesión democrática" (concepto acuñado por Larry Diamond), ha sido imparable en las dos primeras décadas de este siglo. Garton Ash (2021) señala que, a finales de 2020, y por primera vez en este siglo, si se toman los países con más de un millón de habitantes, hay menos democracias que regímenes no democráticos.

La conclusión desde la perspectiva de la Educación no es muy alentadora: si hasta 2010 hubiera sido fácil mostrar evidencia sobre una relación clara entre la universalización de la educación y el progreso de la democracia, la relación parecería haberse dado la vuelta en la segunda década de este siglo. Dicho de otra forma, a pesar del enorme progreso en construir sistemas escolares cada vez más inclusivos, y de haber invertido masivamente en la Educación como bien público a nivel global, nacional y local, la recesión democrática ha avanzado rápidamente, tanto que 2016 podría considerarse el punto de inflexión: en Estados Unidos y Gran Bretaña, las dos democracias más antiguas del globo, ganan Trump y el Brexit, 
respectivamente. La catástrofe educativa es evidente: una educación universal - y en el caso de estos dos países de una calidad muy por encima de la media - no solo no evita la recesión democrática, sino que, al menos en cierta medida, podría incluso ponerse a su servicio.

No se trata ya, por tanto, de lectura comprensiva o de otras habilidades instrumentales básicas. El esfuerzo educativo por desarrollar valores democráticos, impulsar el aprendizaje de la convivencia pacífica, el respeto al diferente y al discrepante, tampoco está produciendo los resultados esperados en una mayoría cada vez más abrumadora de países.

No pretendo ni mucho menos sugerir aquí que la universalización de la educación esté dando alas a la recesión democrática (aunque tal vez algunos lo suscribirían). Es en todo caso al contrario: la recesión democrática ataca a la raíz de la educación universal, que no es otra cosa que la democratización del aprendizaje. No obstante, la recesión democrática sí podría estar acelerando la devaluación y debilidad políticas que son tradicionales al sector educativo, y ello justamente al hilo de la expansión y la universalización de la educación que, precisamente, siempre han sido miradas con recelo o directamente con rechazo desde posiciones antidemocráticas. Así, con todo y que existe evidencia empírica de que una buena educación para todos es tan posible como deseable, el escepticismo y hasta la resistencia tanto activa como pasiva a la universalización de la educación se manifiestan con frecuencia en la opinión pública global, se reflejan con claridad en las opiniones y actitudes de buena parte del profesorado (Fernández Enguita, 2009) y hasta en lo que conocemos como desafección o desenganche escolar y creación de culturas anti-escuela entre los estudiantes (HBSC, 2020).

España podría presentarse como uno de los ejemplos destacados de este fenómeno de la devaluación política de la escolarización universal. Desde hace al menos tres décadas, intelectuales progresistas muy destacados y con gran influencia en medios de comunicación de prestigio parecerían coincidir con otros claramente reaccionarios en que hay una gran conspiración de políticos y pedagogos que ha destruido la educación "a fin de gobernar con mayor facilidad". Curiosamente, también en Francia, la cuna de la Ilustración y de la escuela pública laica, se ha planteado -con considerable ruido mediático en la última década- la idea de que existe un "pacto inmoral" desde finales de los 80 entre políticos y pedagogos (la nomenclatura ministerial y los pedagogos sesentayochistas) "que ha malvendido las exigencias académicas con el pretexto del igualitarismo". Tal pacto interesaría sobre todo a las elites, que continuarían beneficiándose del mantenimiento de una doble red educativa pública, una para ellos y otra para las masas (Coignard, 2010). La similitud de este debate en ambos países es sin duda notable. Más allá de conspiraciones, hoy tristemente más de moda que nunca, la devaluación política y mediática de la educación es indudable y tiene su origen en el agnosticismo, más o menos manifiesto, más o menos declarado o admitido, de unos y otros sobre la universalización de la educación.

\subsection{La imparable ascensión de la educación en la sombra}

Mark Bray acunó a comienzos de siglo el concepto de shadow education para denominar al conjunto de clases y tutorías particulares impartidas por profesores individuales, por academias de preparación de exámenes y, más recientemente, por empresas que ofrecen servicios similares online. En el Reino Unido, el uso del término shadow en este caso no solo sugiere que se trata de un sector predominantemente oculto (el caso de las clases particulares) sino también que, en cierto modo, compite con la escolarización propiamente dicha ${ }^{2}$. El tamaño de este sector es, por su propia naturaleza semi-oculta, difícil de establecer

1 Marías, J. (2021, 17 de enero). Que no sepan, no se expresen, no piensen. El País. El artículo tiene una visión ya no catastrófica sino apocalíptica sobre la educación en España. Merecería una reflexión seria que un escritor de su talla, candidato sólido al Nobel, publique algo así. Pero la cita que encuentro más reveladora y con mayor enjundia entre las muchas de este cariz es de Félix de Azúa y no resisto la tentación de traerla también a esta nota: "Los lamentos sobre el estado de la educación en las naciones supuestamente educadas son un plañido estéril. Nadie hará nada para remediar el regreso al analfabetismo masivo porque nadie sabe cómo evitarlo, ni (lo que es más interesante) para qué evitarlo. No creo demostrable que el mundo de pasado mañana exija más universidades. Sí, en cambio, más academias de artes marciales” publicado en El País, a 10 de enero de 2005, p. 15.

2 Así, en la tradición política británica, shadow government o gobierno en la sombra se refiere al gabinete ministerial paralelo formado por el partido de la oposición, en el que se designa a una persona como "sombra" de cada ministro del gobierno. En este sentido, shadow education implica la existencia de un servicio educativo 
o aun de estimar con una cierta precisión. Sin embargo, sobre todo en aquellos países donde existen exámenes públicos muy selectivos y competitivos -tanto para la graduación como para el acceso al siguiente nivel educativo o para la entrada en escuelas especiales de elite, usualmente ya en el nivel universitario-, las clases particulares y la industria de la preparación de exámenes han crecido de un modo tan formidable (obviamente, a medida que aumentaba la tasa de matrícula en primaria y secundaria) que es cada vez más frecuente que las encuestas de hogares incluyan preguntas específicas sobre gasto familiar en clases particulares y en otras formas de educación "suplementaria".

Así, en países como Egipto, Corea del Sur, India, China y muchos otros, el volumen de gasto privado en esta educación en la sombra compite con el de gasto público en escolarización y, en los casos más extremos, puede afirmarse que supone ya una privatización de facto del sistema educativo público (Sohby, 2012). Quede claro que no se trata aquí del crecimiento del sector educativo privado, ni de la financiación pública del sector privado o de la gestión privada del sector educativo público, sino de una privatización desde dentro del sistema público, es decir, llevada a cabo principalmente por sus propios funcionarios, con lo que la educación en la sombra entra de lleno en el territorio de la corrupción ${ }^{3}$. Cabría usar aquí la metáfora del caballo de Troya si no fuera porque las tutorías y clases particulares no son precisamente un regalo.

Mientras que el gasto público en educación se frenó radicalmente durante la Gran Recesión y se enfrenta en esta década a importantes restricciones por causa de la pandemia, el gasto privado en tutorías privadas continúa creciendo a gran velocidad. En 2017, las estimaciones de GSV (2018) que recogían fundamentalmente los datos de grandes empresas dedicadas a la preparación de test y exámenes cifraban este mercado en unos 100 mil millones de dólares, siendo el rubro que más crecía en el sector de la educación junto con el de las inversiones en tecnología educativa relacionadas con el desarrollo del aprendizaje en línea ${ }^{4}$. Estimaciones más recientes y proyecciones de futuro, en este caso de ReportLinker (2020) -un think-tank que hace seguimiento a que llama "industria global de las tutorías privadas"-indican que la que hemos llamado educación en la sombra alcanzará los 218 mil millones de dólares en $2027^{5}$.

La pandemia del COVID 19 también está acelerando la consolidación como fenómeno global (hace tiempo que no puede hablarse ya de su simple surgimiento) de este mercado privado construido sobre la plataforma de la educación pública. El cierre de escuelas durante meses, y los modelos híbridos de escolarización forzados por la evolución de la pandemia han desencadenado una demanda todavía mayor de servicios de tutoría privada en línea, y ahora ya ni siquiera tan relacionada con la preparación de exámenes externos -aunque ésta sigue siendo su principal generador- sino también con asegurar lo que durante la pandemia se ha dado en llamar continuidad en el aprendizaje de los estudiantes. En los primeros meses de la pandemia, fueron bastantes los países -Francia e Italia con el Bac y la Maturità, respectivamente, son ejemplos muy destacados- que optaron por suspender la celebración de esos exámenes con la

paralelo al público que mimetiza su organización y su curriculum, y que responde a las mismas demandas del alumnado y sus familias.

3 En Egipto, por poner uno de los casos nacionales más extremos, la enorme demanda de las familias por estos servicios se une a los bajos salarios del personal de la administración educativa y de los propios docentes para crear un mercado inmenso, especialmente con los estudiantes de los tres últimos años de secundaria de cara a la preparación del legendario examen de graduación y acceso a la universidad (el Thanwiyya Amma). Los miembros de los comités que preparan las preguntas del examen consiguen sobresueldos muy importantes ofreciendo en este mercado "ejercicios especializados" para preparar el examen. Muchos autores de libros de texto producen dos versiones de sus libros, una para la distribución generalizada y otra, de mucha mayor calidad y mejor orientada a la preparación del examen, que publican y venden a través de editoriales privadas. Pero incluso el profesorado en el sistema público ofrece lecciones privadas a sus propios alumnos, para las que se reserva el material más útil de cara al examen, lo que ha terminado llevando a que, en esos dos o tres últimos cursos de la secundaria, las aulas estén casi vacías porque los estudiantes prefieren asistir a las clases particulares que a sus centros (ver, entre otros, Assad y Kraft, 2015). El actual Ministro de Educación se ha propuesto eliminar este examen -que tiene más de un siglo de tradición- en lo que podría ser la reforma educativa de más calado y alcance en la historia del país.

$4 \mathrm{Si}$ bien es evidente que hay un solapamiento cada vez mayor entre ambos dado que la oferta de tutorías privadas es cada vez más también por internet.

5 Para poner la cifra en perspectiva, es aproximadamente el PIB de Portugal de 2019. 
idea de evitar una fuerte crisis de equidad en el sistema educativo. Pero los exámenes regresaron en todas partes y, si el crecimiento rampante de la educación en la sombra ya suponía una incesante catástrofe para la equidad y la calidad de la educación pública, su expansión a través de servicios de internet podría llevarnos a escenarios aún más extremos, a menos que se lleve a cabo una revisión profunda de las políticas de exámenes públicos y de las certificaciones y asignación de oportunidades con ellos relacionadas.

\section{Los elementos contra los que ha de luchar la innovación en educación}

\subsection{Desigualdad y segregación siguen siendo el ADN de los sistemas educativos}

La paradoja del desarrollo de la educación en las últimas dos décadas es que, cuanto mayor es la igualdad en el acceso a la escolarización, mayor es también la inequidad del aprendizaje -en definitiva, de la calidad- entre los estudiantes. El riesgo de una mayor desigualdad de aprendizaje a medida que se expande el sistema educativo crece porque la proporción de los excluidos del aprendizaje no se reduce tanto ni tan rápido como sería necesario a pesar de las sucesivas olas de reformas e innovaciones. Por otra parte, el riesgo de que la desigualdad siga aumentando (en términos de pobreza de aprendizaje, como se sugería más arriba) está también en que las innovaciones tienen a suponer inversiones considerables en los países ricos y operaciones más bien low-cost en los países en desarrollo. Cierto que la innovación no tiene por qué ser cara, pero la cuestión de la desigualdad sigue estando presente en el elitismo intrínseco a muchas innovaciones de éxito (programas piloto, centros de excelencia, etc.) que rara vez pueden ser escaladas y beneficiar a más de unos pocos.

La llamada crisis del aprendizaje persiste, por tanto, no ya por la incompetencia, o la corrupción, de gobiernos y administraciones ni por la insuficiente financiación sino, sobre todo, por la desigualdad y la cultura de segregación que sigue prevaleciendo en los sistemas escolares. Hay una contradicción flagrante entre el espíritu de las leyes educativas, las retóricas de la inclusión y de la democratización del aprendizaje, y las prácticas cotidianas que mantienen o incluso refuerzan la segregación de escuelas y sistemas enteros. Muchos reformadores e innovadores tienen a obviar que desigualdad y segregación forman parte de la llamada gramática de la escuela y que las soluciones no pueden ser por tanto solo cuestión de gobernanza o financiación.

La desigualdad educativa y su corolario practico, la segregación, se han explicado hasta ahora por el carácter de bien escaso tanto de la educación de calidad como del empleo de calidad. Sin dejar de ser válida dicha explicación, a pesar del empuje de una educación universal de calidad, surge con fuerza un nuevo desafío para la igualdad en educación, esta vez desde las políticas identitarias. La actual carrera entre identidad e igualdad educativa conduce a nuevas formas que podría adoptar la segregación en tanto en cuanto todo tipo de colectivos se muestran dispuestos a sacrificar igualdad por identidad, es decir, a segregarse para distinguirse. Ya no es solo la identidad nacional, que por otra parte está en el origen de los sistemas públicos de educación, sino las múltiples identidades que se presentan hoy como medida de la dignidad humana diferencial y que, casi siempre, son identidades en conflicto y hoy susceptibles de "autodeterminación” y hasta de supuestos derechos colectivos: etnia, orientación sexual, lengua materna, ideología y adscripción partidista, religión, dieta alimenticia, etc. Estas políticas del sentimiento, que lo son tanto o más también del resentimiento, pueden estar convirtiéndose en otro motor de desigualdad y segregación, esta vez además con el soporte y el marchamo del pensamiento político supuestamente más progresista.

La pregunta para los educadores y para los sistemas educativos sería la siguiente: ¿Es posible, y deseable, educar para construir identidades múltiples articuladas sobre el principio de la ciudadanía global? ¿Cabe una ciudadanía sin apellidos y con derechos individuales o estamos más bien dispuestos a asumir un cierto volumen de desigualdad a cambio del mayor reconocimiento de las diferencias y de los derechos colectivos? La dinámica de narcisismo y victimismo, que se alimentan mutuamente en la construcción de identidades en conflicto, es en buena medida el combustible que utilizan quienes hacen avanzar hoy la recesión democrática; y supone tal vez el reto educativo de mayor alcance y profundidad que afronta nuestro siglo. 


\subsection{La confianza social sobre la educación descansa más sobre la naturaleza competi- tiva y diferenciadora del sistema que sobre su potencial igualador}

No son pocos los países en los que a los Ministerios de Educación se les llama coloquialmente Ministerios de Exámenes. Se trata de exámenes de graduación y/o de acceso al siguiente nivel educativo que, hasta hace un par de generaciones, solo hacían una minoría de estudiantes, sobre todo en el mundo en desarro1lo. Pero la universalización de la educación primaria y el gigantesco crecimiento del acceso a la secundaria y a la educación superior, han puesto a los exámenes externos en el centro del tablero educativo. Para los millones de familias de todo el mundo que invierten cantidades enormes en tutorías privadas, la medida del éxito escolar no es tanto el aprendizaje conseguido como las calificaciones que sus hijos obtienen en dichos exámenes y la posesión de los certificados, diplomas y oportunidades que tales calificaciones les confieren. Las familias estarían pues más preocupadas por el retorno privado de la educación -el diferencial de lo que ganan y ganarán sus hijos- que por el retorno público -la renta educativa básica que asegure la inclusión y la igualdad de oportunidades-.

La universalización del acceso a la educación ha venido pues acompañada de distintos grados de credencialismo en función de la naturaleza del sistema de exámenes externos en cada país y de los incentivos, sean buenos o perversos, que crean en la sociedad y en la comunidad educativa. Procede preguntarse, en general y en cada país, dónde está la línea que separa la democratización del credencialismo, y el papel que juegan los exámenes externos tendría mucho peso en la respuesta. Cuanto mayor sea la brecha entre aprendizaje y certificados, entre competencias y diplomas, habrá menor democratización y mayor credencialismo. Y esto implica también, obviamente, menor calidad y equidad.

Un ámbito tan central como crucial para reformas e innovaciones es el curriculum escolar en el nuevo contexto de la universalización de la educación. Reformas e innovaciones curriculares han de llevar necesariamente aparejadas propuestas consistentes de reforma de los exámenes externos y de la evaluación del aprendizaje de los estudiantes en general. Especialmente en los países en desarrollo, donde a pesar de su reciente expansión, los sistemas escolares parecen seguir diseñados para una elite, tanto curriculum como exámenes, y la consistencia, o inconsistencia, entre ambos, han cambiado muy poco en las últimas décadas. Muchos esfuerzos renovadores en materia curricular fracasan o no pueden sostener su éxito porque el inmovilismo y los incentivos que crean los exámenes externos continúa amparando ese foco en la elite, que conlleva la fuerte inercia de currículos enciclopédicos y modelos de evaluación que producen altas tasas de fracaso y repetición.

Puede traerse aquí a colación el debate sobre el curriculum basado en competencias como el esfuerzo contemporáneo más importante para transformar un curriculum elitista en otro más inclusivo. Son muchos quienes contemplan con sano y justificado escepticismo el curriculum competencial: para algunos, supone una devaluación y adulteración de la educación que no hará sino que siga bajando el nivel y aumentando la ignorancia de las nuevas generaciones; para otros, sería un curriculum de segunda para las masas y al servicio exclusivo del mercado laboral (no en vano la noción de competencia como organizador curricular procede de la Formación Profesional), mientras que el conocimiento valioso seguiría solo a disposición de una elite (Weelahan, 2007). Sin embargo, un curriculum basado en competencias tiene un claro potencial de superar y dejar atrás el elitismo de los curricula y los exámenes actuales. Contribuiría a cerrar la brecha entre aprendizaje y certificación y, con ello, a disminuir el credencialismo y profundizar la democratización del sistema. ¿Qué curriculum y qué evaluación defienden mejor los intereses a largo plazo de todos los estudiantes y consiguen de verdad mejor calidad y más igualdad? Esa es al fin y al cabo la cuestión que reformadores e innovadores, y por supuesto, investigadores tienen que contestar.

\subsection{La erosión del contrato educativo y el fin de la meritocracia}

Podría llamarse contrato educativo a una parte integrante del más amplio contrato social. Básicamente, se trata del principio en el que hemos sido socializados generaciones de estudiantes: "si vas a la escuela cada día, estudias seriamente y te gradúas, tendrás un buen futuro". Una vez más, la universalización de la educación básica ha hecho crecer las expectativas y aspiraciones legítimas, basadas en ese contrato, de quienes se han ido incorporando al sistema educativo. Pero el credencialismo y la brecha entre aprendizaje y diplomas han ido cambiando las reglas de juego de la transición desde la educación al empleo, con el resultado de que la explosión de aspiraciones se ha visto en buena medida frustrada. El contrato educativo sufre entonces una fuerte erosión porque, como mínimo, certificados y diplomas han pasado 
de ser condición suficiente para ese buen futuro personal a ser solo condición necesaria, pero en modo alguno suficiente. Dicho de otro modo, la crisis del empleo, que es mucho más aguda y profunda entre los jóvenes graduados y en los países en desarrollo, afecta negativamente al valor que la sociedad atribuye a la educación (o, al menos, a los certificados, títulos y diplomas que otorga).

Por otra parte, el ideal meritocrático, que podría considerarse el espíritu que subyace al contrato educativo, ha entrado en crisis desde muchos frentes. Por una parte, se acumula la evidencia de que el mérito académico está mucho más relacionado con la cuna de lo que habíamos creído; y que distribuir oportunidades y empleos en función del mérito no reduce la desigualdad ni crea ese "terreno de juego equilibrado" que se había soñado (Sandel, 2020). De hecho, la meritocracia vendría a desempeñar una función legitimadora de la desigualdad y hasta contribuiría a incrementarla. Por otra parte, las políticas identitarias hoy en alza también están desafiando a la meritocracia, reclamando nuevos criterios de asignación de oportunidades en función de identidades diferenciales que solo así podrían ser reconocidas y respetadas. En definitiva, la propia adquisición del mérito podría ser considerada dudosa o incluso ilegítima.

En educación, si se descarta la meritocracia, y con ella el elitismo de curricula y exámenes al que me refería más arriba, el problema es que no hay paraíso al que regresar y tampoco hay mucha claridad sobre posibles paraísos alternativos hacia los que ir (por ejemplo, basados en lo identitario). El reto en este caso está en cómo asegurar la inclusión y la igualdad educativas sabiendo que el contrato educativo tradicional ya no está funcionando (o lo hace solo para una minoría) en el contexto de una educación universal. ¿Es necesario por tanto proponer un nuevo contrato educativo a los estudiantes y a sus familias?

\subsection{La industria global de la posverdad y los hechos alternativos ha emergido como una suerte de sistema educativo también alternativo}

La hoy llamada posverdad y el bello eufemismo de hechos alternativos para denominar lo que antes se conocía por mentira o bulo han existido siempre. La novedad contemporánea es que ahora se trata de una industria global, es decir, de todo un sector de actividad económica que emplea a miles de personas en todo el mundo, tanto en el sector público como en el privado, y que opera fundamentalmente en internet. Esta industria global para la fabricación y diseminación de mentiras al servicio de poderes establecidos (Estados y empresas) o emergentes (sectas, lobbies, etc.) está en la raíz de la recesión de la democracia que hoy sufrimos. Su objetivo parecería ser devolver al mundo a la Edad Media y su modus operandi es dinamitar la base de la convivencia en democracia socavando la relación de confianza de los ciudadanos con las instituciones públicas, incluidos sus gobiernos, los medios de comunicación y, por supuesto, los centros educativos. No procede ver aquí conspiración alguna, dado que esta industria global opera cada vez más a la luz del día y, en muchos casos, financiada voluntariamente por los propios ciudadanos que consumen sus productos. Muchos Estados y organizaciones supranacionales están de hecho tratando de regular legalmente una defensa democrática ante las actividades de la industria de la posverdad.

Dado que esta industria compite sin complejos con los sistemas educativos formales, la devaluación política de la educación pública la convierte en un espacio preferente y hasta prioritario para la actividad de aquélla. Cuadra bien en este contexto el mensaje machacón de que las escuelas públicas son ineficientes y de mala calidad por definición. Y todavía mejor el de que las administraciones educativas siempre se equivocan y nada les sale bien, siendo su torpeza y capacidad para cometer errores no solo proverbiales sino ilimitadas; con excepción, eso sí, de cuando lo que se proponen es malo para nosotros: entonces su eficacia se torna extraordinaria y actúan con el cálculo y la brillantez de artista consumado. Para el desmantelamiento de la confianza de los ciudadanos en las instituciones, esta ley de la perversidad intrínseca de las administraciones públicas es crucial: son tan ineficaces para lo que nos gusta como eficaces para lo que no nos gusta (y el "nos" puede ser cualquiera; somos todos).

Noticias falsas, hechos alternativos y sesgos de todo tipo sobre la educación solo pueden contrarrestarse con información y datos de calidad, con máxima transparencia por parte de centros y de administraciones y, en definitiva, con más y mejor educación. La información pública disponible y la transparencia como principio de conducta institucional contribuyen decisivamente a empoderar a los más vulnerables y desfavorecidos y a avanzar por tanto la agenda de la igualdad. La pobreza en educación es la dimensión menos visible de la pobreza, así que información y transparencia son cruciales primero para hacerla más visible y después para que quienes la sufren quieran y puedan salir de ella. 
Por otra parte, no obstante, parece igual de cierto que la industria de la posverdad está consiguiendo que la disponibilidad de información, incluida la evidencia empírica, ni aclare el debate público ni mucho menos reduzca la creciente polarización. De hecho, por ejemplo, muchos defensores de la igualdad miran con recelo la publicación de datos sobre el desempeño de centros educativos y de los estudiantes por los usos perversos que potencialmente pueden hacer de esos datos tanto los medios de comunicación como las propias administraciones; usos perversos que podrían en según qué casos justificar e incluso profundizar la desigualdad. Existe pues un dilema mayor entre opacidad para evitar malos usos de la información y transparencia para cerrar la brecha de información entre ricos y pobres en educación. Representa sin duda uno de los elementos contra los que debe luchar hoy la innovación educativa. El reto está en que la calidad de la información y la capacidad de comunicarla eficazmente desde el sector educativo serían el mejor antídoto contra su manipulación.

\subsection{La búsqueda de consenso en educación no tiene muchos valedores y para que la educación pública progrese sostenidamente hace falta consenso}

Si el sector educativo es en estos tiempos terreno abonado para las prácticas de la industria global de la posverdad y de las políticas identitarias, la construcción de consensos para reformas duraderas e innovaciones sostenibles, que no ha sido nunca tarea fácil, lo va a ser aún menos ahora. Si el sesgo de confirmación campea triunfador en el contexto de alta polarización en que nos encontramos, todo aquello que pueda unir estará bajo sospecha, y solo lo que divide parece dar dividendos políticos. Incluso el propio concepto de consenso puede verse como sospechoso, bien por ser el reflejo de una conspiración -recuérdese por ejemplo el "pacto inmoral" en Francia-, bien por ser expresión de frentismo, es decir, la imposición a toda la sociedad de un pensamiento hegemónico que no defiende los intereses de todos. No parecen existir muchos incentivos para grandes pactos en materia de educación ${ }^{6}$, lo que refuerza y agudiza el problema de falta de liderazgo político en el sector, un vacío que a duras penas tratan de llenar o compensar quienes podríamos denominar innovadores locales, es decir, los líderes pedagógicos en los centros y distritos escolares.

Sin embargo, sabemos bien que las políticas de reforma e innovación que pueden aspirar a generar cambios profundos y sostenibles son más maratón que carrera de velocidad, requieren por tanto visión a largo plazo, liderazgo político más allá de cambios de gobierno, financiación predecible y confianza pública en los profesionales. Cómo conseguir consensos sólidos en educación cuando a buena parte de los poderes $\mathrm{y}$ de los actores no parece interesarles es otro de los grandes retos en nuestro sector. Por eso, dicho sea de paso, han sido tan importantes los objetivos de desarrollo del milenio a principios de siglo y, en este momento, el cuarto objetivo de desarrollo sostenible para 2030. Son consensos que funcionan como un bien público global.

\section{6. ¿Apocalipsis ahora?}

Una posible conclusión general a estos cinco grupos de problemas y tendencias con los que he tratado de dar sentido a las llamadas catástrofes de la educación es que el gran proyecto ilustrado de la educación pública universal, formulado hace dos siglos y medio, estaría atravesando hoy su peor crisis de crecimiento, justamente cuando más cerca está de conseguir sus objetivos fundacionales. Los problemas son sin duda de gran calado, y quienes podríamos llamar abiertamente enemigos del proyecto ilustrado son muchos y atacan desde muy variadas posiciones. Por si esto fuera poco, la pandemia del COVID-19 está acelerando y exacerbando los efectos negativos de todos y cada uno de estos problemas, empezando especialmente por el de la desigualdad (Long et al., 2020).

Aunque sería tentador dejarse seducir por esta idea de una tormenta perfecta contra el proyecto de la Ilustración, y si bien no deja de ser una hipótesis razonable, mi intención aquí ha sido identificar las catástrofes, pero sin incurrir en el catastrofismo. Como sugiere Steven Pinker en la cita que encabeza este artículo, tan imprescindible como diagnosticar los problemas es reconocer el progreso que se ha logrado. Obviar, olvidar o minimizar ese progreso tiene el efecto de magnificar los problemas y puede dar lugar a propuestas de "derribar las instituciones y empoderar a un líder que restablecerá la grandeza" ... o, podríamos añadir, que nos traerá la grandeza que hasta ahora se nos había hurtado.

6 El caso particular de España a este respecto lo analizamos en detalle en Gortazar y Moreno (2017). 
El progreso de la educación universal de calidad para todos a lo largo de los últimos dos siglos es tan extraordinario como innegable. Ese progreso es justamente el que permite aspirar a conseguir objetivos aún más ambiciosos y en menos tiempo, lo que a su vez da lugar a mayores expectativas personales, familiares y sociales sobre el sistema educativo. Así, cuanto más inclusivo y eficiente es el sistema educativo, mayores y más radicales son las demandas que se hacen sobre él y más visibles son sus debilidades. Así que es precisamente ese progreso el que hace cada vez menos aceptable todo aquello que aún queda por conseguir o, mucho más aún, todo retroceso que se pueda producir. No cabe duda de que estamos atravesando un serio retroceso, en este caso acelerado por las consecuencias de una pandemia, y que resulta necesario hacer frente a nuevos problemas y revisar viejos contratos en educación. Pero, una vez más con Pinker (2018):

No todo problema es una Crisis, una Plaga, una Epidemia o un Amenaza Existencial, y no todo cambio es el Fin de Esto, la Muerte de Aquello o el Amanecer de una Era Pos-Algo. No confundas el pesimismo con la profundidad: los problemas son inevitables, pero los problemas son solubles, y diagnosticar todo contratiempo como un síntoma de una sociedad enferma es un intento rastrero de aparentar seriedad. (p. 548)

\section{Una agenda global para la innovación educativa}

La conocida como Ley Sevareid es una referencia habitual en muchos campos, incluida la Agricultura, la Química o el análisis de complejidad de sistemas en Informática. Dicha ley establece que "la principal causa de los problemas son las soluciones". En políticas públicas, empezando por políticas sociales como la educación, la Ley Sevareid resulta útil porque, primero, las soluciones de hoy serán por definición los problemas de mañana; y segundo, porque en según qué casos, se buscan soluciones para problemas que en realidad no existían o para los que, como reza el proverbio castellano, el remedio puede ser mucho peor que la enfermedad. En otras palabras, la innovación en educación no solo lucha contra los elementos, sino que, en muchos casos, podría estar luchando también contra sí misma. Considerando la fuerte presión por reformar e innovar bajo la que está el sector educativo, la búsqueda de soluciones eficaces y más o menos mágicas se enfrenta además a la tozudez de una realidad que no admite soluciones de talla única y que advierte en contra de todo tipo de ortodoxia reformadora o de hojas de ruta estándar. Aun así, sigue siendo obligado saber qué funciona y qué no, en qué circunstancias, y con cuánta seguridad puede afirmarse; en definitiva, qué soluciones son las que resuelven más problemas de los que crean. Y es que, además de grandes ideas y buenas intenciones, es indispensable la evidencia sólida, y más ahora que la evidencia científica goza de tan mala prensa, sobre cómo mejora, o no, el bienestar educativo de la sociedad y, por tanto, sobre cuál es la mejor manera, en cada lugar y en cada momento, de gastar el siempre escaso dinero disponible para innovar.

Entre las lecciones aprendidas sobre innovación en el sector educativo, habitualmente atacado por reformar mucho y cambiar poco, hay dos que son cruciales: una es que el principal desafío práctico está en cómo escalar, es decir, cómo generalizar e institucionalizar esas buenas ideas, intervenciones, programas y políticas. La dificultad no está solo en cómo escalar el tamaño, cómo llegar a todo el sistema educativo, sino en cómo escalar también el impacto a medida que se escala el tamaño. Escalar tamaño e impacto de las innovaciones equivale de hecho a democratizar el aprendizaje, y esto implica que las innovaciones que marcan la diferencia se conviertan en instituciones en el sentido amplio de ese concepto. La pregunta clave es pues cómo hacer disponible para todos lo que hasta ahora solo lo había estado para una minoría o para una elite. Por tanto, el desafío de escalar no es solo una cuestión de músculo implementador, logística y soluciones tecnocráticas; es sobre todo cuestión de desarrollo profesional, institucional y de economía política.

La segunda lección es que, a pesar de que el consenso más fácil de construir en educación es para invertir más en personal, en equipamiento y en materiales, todo ese gasto por sí mismo no garantiza mejora alguna ni en calidad ni en equidad. Esto no quiere decir que haya que gastar menos; al contrario, otro reto clave para las políticas de innovación está justamente en la financiación, y es el de cómo incrementar de modo sostenible el espacio fiscal dedicado a mejorar la educación. 
Proponer una agenda global para la innovación en educación, centrada en este caso en los países en desarrollo (donde se concentra la pobreza en educación), no es por tanto tarea fácil, pero es desde luego imprescindible. Las agencias de desarrollo, bilaterales y multilaterales, las fundaciones y ONGs, y muchos otros actores que invierten miles de millones de euros en la educación de estos países necesitan con urgencia esa evidencia para no malgastar sus recursos en intervenciones que no pasen de ser una moda, que resulten infructuosas en términos de calidad y equidad educativa o, aún peor, que sirvan a intereses ajenos al aprendizaje de los estudiantes de esos países.

De hecho, la evidencia disponible es cada vez más abundante y sofisticada. Dos trabajos recientes, uno de carácter más cualitativo (Perlman y Winthrop, 2016) y otro más cuantitativo (Global Education Evidence Advisory Panel, 2020), son de gran utilidad. El primero identifica 14 grandes proyectos innovadores en el mundo en desarrollo y se centra en cómo pasaron con éxito de una fase piloto a involucrar a millones de estudiantes. Además, todos ellos son de "abajo-arriba”, es decir, iniciativas de innovadores locales, surgidas sin mayor apoyo de las administraciones educativas y, en algún caso, a pesar de ellas. El segundo reúne la evidencia procedente de más de 150 evaluaciones de impacto en 46 países distintos, y se fija en la relación de coste eficacia de una selección de intervenciones y políticas innovadoras de "arriba-abajo", esto es, impulsadas por administraciones educativas ${ }^{7}$. Es posible complementar asílas aportaciones desde una visión de la innovación educativa como proceso orgánico que solo puede surgir desde la práctica y los prácticos, con otra visión, para la que el cambio sistémico no solo es posible, sino que es además responsabilidad ineludible de gobiernos y administraciones.

El primer trabajo, titulado Millions Learning, concluye que el éxito de los 14 proyectos locales pasaba primero por su foco en el aprendizaje y por la adaptación flexible al contexto y las necesidades locales. Pero, una vez iniciado su camino, y con la vista puesta en su generalización (escalar), todos tienen en común una serie de factores que constituyen toda una agenda para la innovación en la práctica. Destaco las siguientes:

- Crear alianzas por la educación. La construcción de consenso marca la diferencia, no solo por los beneficios del apoyo político sino también por aumentar la confianza pública sobre el sector educativo.

- Desarrollar cultura de investigación y desarrollo en los centros y entre el profesorado, impulsando también la confianza pública en la profesión docente.

- Identificar líderes comprometidos con el aprendizaje, tanto innovadores locales como personas relevantes en la comunidad/sociedad, pero ajenas al sistema educativo.

- Financiación predecible, especialmente en la transición de la fase piloto a gran escala.

- Disponibilidad de datos confiables sobre el aprendizaje de los estudiantes: deben considerarse un bien público y usarse para seguir promoviendo una cultura profesional de la experimentación y la mejora.

El informe del Global Education Evidence Advisory Panel (un grupo creado en 2020 con representantes de organismos y agencias internacionales y con investigadores independientes) trata de responder a la pregunta básica de qué innovación va a marcar mayor diferencia sobre el aprendizaje de los estudiantes y al menor coste. El análisis se lleva acabo usando el concepto de años de escolarización "netos en aprendizaje", es decir, tiempo de escolarización, ajustado por la calidad del aprendizaje adquirido. Cantidad y calidad de la escolarización se unen así en una sola métrica de progreso. La Figura 1, que resume parte de los resultados, muestra cómo la innovación que añade más valor al aprendizaje por cada euro gastado es, precisamente, ofrecer a la sociedad y a los actores clave de la comunidad educativa información completa y detallada sobre calidad, beneficios y coste del sistema escolar. La transparencia sale pues a cuenta, pues defiende sobre todo los intereses de quienes están fuera de juego cuando la información clave sobre la educación no está disponible o no es fácilmente asequible para todos.

7 Muy relevante también en este sentido es el trabajo de la Education Endowment Foundation (Reino Unido), que reúne una detallada lista de intervenciones innovadoras y la ordena por impacto, solidez de la evidencia disponible y coste de implementación. https://educationendowmentfoundation.org.uk/evidence-summaries/teachinglearning-toolkit/\#closeSignup 
Figura 1

Años de escolarización netos en aprendizaje (LAYS) ganados por \$100, por categoría

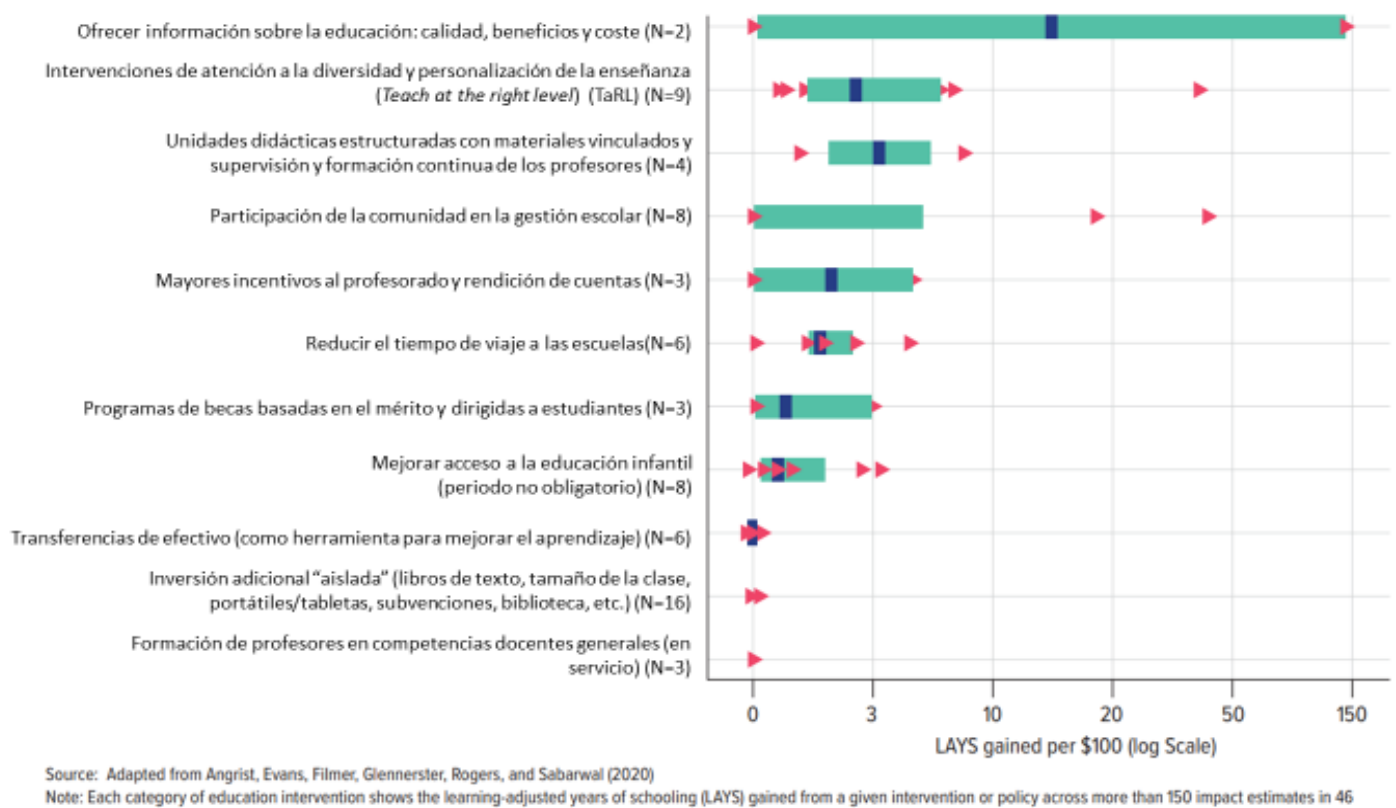

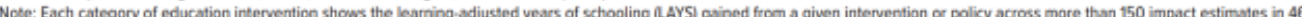
countries. Study categories are ranked by mean impact, from highest to lowest The shaded boxplot delineates the 25 th and 75 th percentles. The y-axis is reported on a natural log scale.

Nota. Adaptado de Angrist y otros (2020). Cada categoría de intervención educativa muestra los años de escolarización netos en aprendizaje ganados por los estudiantes a partir de más de 150 evaluaciones de impacto en 46 países. Las categorías estudiadas aparecen ordenadas por su impacto promedio, de más alto a más bajo. El diagrama de caja sombreado marca los percentiles 25 y 75 . El eje y aparece en una escala logarítmica.

A continuación, el análisis confirma el impacto positivo de intervenciones que buscan mayor personalización de la enseñanza, lo que incluye agrupamientos flexibles y adaptaciones curriculares de todo tipo. En este sentido, es interesante que algunos países desarrollados están promoviendo tutorías individualizadas para combatir la pérdida de aprendizaje causada por el largo cierre de centros educativos, solo que en este caso organizadas y financiadas públicamente (Department for Education, 2021). Por otro lado, el Informe ofrece evidencia contundente de que conceder incentivos monetarios a los estudiantes como fórmula para mejorar el aprendizaje no es una buena inversión. Y lo mismo ocurre, como ya se sugería más arriba, con las inversiones aisladas en materiales, equipamiento y otras intervenciones siempre muy costosas, en especial en sistemas en plena expansión. Se trata de inversiones muy visibles, y que por tanto pueden dar un buen rédito político a las administraciones y/o a los bienintencionados donantes que las financian. Pero el rédito de cara al aprendizaje de los estudiantes es mínimo, y disminuye aún más a medida que el país va resolviendo la cuestión del acceso a la escolarización básica.

La disponibilidad de toda esta evidencia acumulada, su solidez creciente en distintos contextos y condiciones locales, y sobre todo su carácter público y asequible a todos los actores de la comunidad educativa global, han de hacer posible poco a poco que tanto las decisiones sobre innovación que se toman desde la práctica como las que se toman desde la política no solo estén mejor informadas sino también mejor comunicadas y coordinadas.

\section{Agradecimientos}

Agradezco a Ernesto Cuadra, Luis Crouch, Lucas Gortázar y a dos revisores anónimos los comentarios y sugerencias que han permitido mejorar sensiblemente este artículo. Los errores, omisiones e inconsistencias son de mi exclusiva responsabilidad. 


\section{Referencias}

Assad, R. y Krafft, C. (2015). Is free basic education in egypt a reality or a myth? International Journal of Educational Development 45, 16-30.

Azevedo, J. P., Goldemberg, D., Montoya, S., Nayar, R., Rogers, H. Saavedra, J. y Stacy, B. (2021, 3 de mayo). Will every child be able to read by 2030 ? Why eliminating learning poverty will be harder than you think, and what to do about it. World Bank Blogs.

Angrist, N., Evans, D. K., Filmer, D., Glennerster, R., Rogers, H. y Sabarwal, S. (2020). A new micro measure for education interventions: Learning-adjusted years of schooling (LAYS). The World Bank.

Coignard, S. (2010). Le pacte inmoral: Comment ils sacrifient l'education de nos enfants. Albin Michel.

Department for Education. (2021). National tutoring program. https://nationaltutoring.org.uk/

Fernández Enguita, M. (2009). El anti-cándido: todo va mal, pero irá a peor. Alcance y funciones de la visión apocalíptica de la enseñanza por el profesorado. Papeles de Economía Española, 119, 59-68.

Garton Ash, T. (2021, 9 de diciembre). The future of liberalism. Prospect. https://www.prospectmagazine.co.uk/magazine/the-future-of-liberalism-brexit-trump-philosophy

Global Education Evidence Advisory Panel. (2020). Cost-effective approaches to improve global learning. TheWorld Bank.

Gortázar, L. y Moreno, J. M. (2017). Costes y consecuencias de no alcanzar un pacto educativo en España. Revista Educación, Política y Sociedad, 2, 9-37.

GSV. (2018). Education factbook 2018. GSV.

HBSC. (2020) Spotlight on adolescent health and well-being: Findings from the 2017/2018. Health behaviour in schoolaged children survey in Europe and Canada. WHO Regional Office for Europe.

Long, H., Van Dam, A., Fowers, A. y Shapiro, L. (2020, 30 de septiembre). The covid-19 recession is the most unequal in modern U.S. history. The Washington Post.

https://www.washingtonpost.com/graphics/2020/business/coronavirus-recession-equality/

Perlman, J. y Winthrop, R. (2016). Millions learning. Scaling up quality education in developing countries. Center for Universal Education at Brookings.

Pinker, S. (2018). En defensa de la Ilustración. Paidós.

ReportLinker. (2020). Global private tutoring industry. https://www.reportlinker.com/p0552740/Private-Tutoring-Industry.html?utm_source=GNW

Sandel, M. (2020). La tiranía del mérito. Debate.

Sohby, H. (2012). The de-facto privatization of secondary education in Egypt: A study of private tutoring in technical and general schools. Compare: A Journal of Comparative and International Education, 42(1), 47-67.

United Nations. (2020). Policy brief: Education during COVID-19 and beyond. United Nations.

Weelahan, L. (2007). How competency-based training locks the working class out of powerful knowledge: a modified Bernsteinian analysis. British Journal of Sociology of Education, 28(5), 637-651.

\section{Breve CV del autor}

\section{Juan Manuel Moreno}

Especialista Principal de Educación en el Banco Mundial. Ha sido responsable de los proyectos de educación del Banco en Armenia, Azerbaiyán, Bulgaria, Jordania, Líbano, Palestina y Egipto. Entre 2011 y 2017, fue miembro del Consejo Directivo del Instituto Internacional de Planificación Educativa (IIPE) de la UNESCO. Hasta incorporarse al Banco Mundial en 2002, fue Profesor Titular de la Facultad de Educa- 
ción de la UNED. Entre 1999 y 2002 sirvió como Vicerrector de Relaciones Internacionales de esa universidad. Ha publicado extensamente sobre reforma educativa y mejora de la escuela, desarrollo del curriculum y políticas de desarrollo profesional del profesorado. Entre ellos, destacan Expanding Opportunities and Building Competencies for Young People: a New Agenda for Secondary Education, que fue el primer informe del Banco Mundial sobre Educación Secundaria (2005); y Jobs for Shared Prosperity: Time for Action in the Middle East and North Africa, también publicado por el Banco Mundial (2013).

Email: jmmolmedillla@gmail.com

ORCID ID: https://orcid.org/0000-0002-3029-771X 